\title{
Valley polarization in magnetically doped single-layer transition-metal dichalcogenides
}

\author{
Y. C. Cheng, ${ }^{*}$ Q. Y. Zhang, and U. Schwingenschlögl ${ }^{\dagger}$ \\ PSE Division, KAUST, Thuwal 23955-6900, Kingdom of Saudi Arabia
}

(Received 2 December 2013; revised manuscript received 11 April 2014; published 28 April 2014)

\begin{abstract}
We demonstrate that valley polarization can be induced and controlled in semiconducting single-layer transition-metal dichalcogenides by magnetic doping, which is important for spintronics, valleytronics, and photonics devices. As an example, we investigate $\mathrm{Mn}$-doped $\mathrm{MoS}_{2}$ by first-principles calculations. We study how the valley polarization depends on the strength of the spin orbit coupling and the exchange interaction and discuss how it can be controlled by magnetic doping. Valley polarization by magnetic doping is also expected for other honeycomb materials with strong spin orbit coupling and the absence of inversion symmetry.
\end{abstract}

DOI: 10.1103/PhysRevB.89.155429

PACS number(s): 73.20.-r, 75.50.Pp, 75.70.Tj

The fields of electronics and spintronics require an active control and manipulation of the charge and spin degrees of freedom [1]. Valleytronics, on the other hand, is very new field that relies on the property that the conduction/valence bands have two or more minima/maxima at equal energy but different momenta. For valleytronics devices it is necessary to induce valley polarization, i.e., control the number of electrons in each valley, typically by strain [2] or a magnetic field [3,4]. In general, two-dimensional materials have raised a lot of interest both for fundamental and applied reasons. Examples are semiconductor quantum wells [5], noble metal surfaces [6], graphene [7], and topological insulators [8]. Semiconducting single-layer transition metal dichalcogenides $M X_{2}$ with $M=$ Mo, W and $X=\mathrm{S}, \mathrm{Se}, \mathrm{Te}$ and the $D_{3 h}$ point group have caught attention, because they display distinctively different physical properties as compared to their bulk compounds with the $D_{6 h}$ point group. There exists a crossover from an indirect band gap in multilayers to a direct band gap in the single-layer limit [9-12]. In the latter the conduction and valence band edges are located at the $K$ points of the two-dimensional hexagonal Brillouin zone. These two inequivalent valleys constitute a binary index for low energy carriers, which gives rise to a valley Hall effect and valley dependent optical selection rules for interband transitions at the $K$ points [13-15]. It has been demonstrated that optical pumping with circularly polarized light can achieve a dynamic valley polarization in single-layer $\mathrm{MoS}_{2}$ [16-18]. However, from the application point of view an equilibrium valley polarization is desirable, which is the topic of the present paper.

The interplay between spin orbit coupling and ferromagnetism in two-dimensional materials gives rise to a variety of unconventional phenomena, such as the quantum anomalous Hall effect [19-22]. In addition, it recently has been put forward that dichalcogenides doped with magnetic transitionmetal atoms form a promising platform for two-dimensional dilute magnetic semiconductors [23-25]. However, these studies did not take into account the spin orbit coupling, which interconnects the spin and valley physics. We propose in this paper a method to control the valley polarization by magnetic doping. Various possible Mn doping sites in single-layer $\mathrm{MoS}_{2}$ are studied to investigate the influence of the exchange field

\footnotetext{
*Yingchun.Cheng@kaust.edu.sa

†Udo.Schwingenschlog1@kaust.edu.sa
}

on the electronic structure by first-principles calculations. We will argue that the strength of the spin orbit coupling together with the exchange energy determine the valley polarization, which can be inverted by modifying the spin polarization.

We present fully relativistic calculations within density functional theory using the Quantum-ESPRESSO package [26]. Ultrasoft pseudopotentials [27] and the generalized gradient approximation (Perdew-Burke-Ernzerhof parametrization) of the exchange correlation functional are employed. All simulations are carried out for a $4 \times 4$ supercell with $16 \mathrm{Mo}$ and $32 \mathrm{~S}$ atoms, as shown in Figs. 1(a) and 1(b), which is large enough to prevent an artificial dopant-dopant interaction due to the periodic boundary conditions. Figure 1(c) demonstrates that the $K$ and $K^{\prime}$ points of the $1 \times 1$ unit cell are folded onto the $K$ and $K^{\prime}$ points of the $4 \times 4$ supercell, respectively. A $10 \AA$ thick vacuum layer is adopted to avoid artificial interaction because of the periodic boundary conditions. A high cutoff energy of $544 \mathrm{eV}$ and a precise $4 \times 4 \times 1$ mesh for the $k$-point sampling are used. The structural optimization is continued until the residual forces have converged to less than $2.6 \times 10^{-3} \mathrm{eV} / \AA$ and the total energy to less than $1.4 \times 10^{-4} \mathrm{eV}$.

It is known that the properties of dilute magnetic semiconductors depend sensitively on the positions of the magnetic impurities in the host semiconductor [28], and thus on the growth method and conditions [29-33]. We therefore place the Mn atoms with a concentration of $6.25 \%$ at various possible absorbtion $\left(\mathrm{Mn}_{\mathrm{HT}}\right.$ : $\mathrm{Mn}$ on top of the hollow site, $\mathrm{Mn}_{\mathrm{MoT}}$ : $\mathrm{Mn}$ on top of $\mathrm{Mo})$ and substitution $\left(\mathrm{Mn}_{\mathrm{Mo}}\right.$ : Mo substituted, $\mathrm{Mn}_{\mathrm{S}}$ : S substituted) sites in single-layer $\mathrm{MoS}_{2}$ and compare the formation energies $\Delta H_{f}=E_{\mathrm{tot}}^{\mathrm{d}}-E_{\mathrm{tot}}-\sum_{i} n_{i} \mu_{i}$, where $E_{\text {tot }}^{\mathrm{d}}$ and $E_{\text {tot }}$ are the total energies of the defective and ideal supercells, $\mu_{i}$ is the chemical potential for species $i$, and $n_{i}$ is the number of atoms added to or removed from the supercell. We have (i) $\mu_{\mathrm{Mo}}+2 \mu_{\mathrm{S}}=E\left(\mathrm{MoS}_{2}\right)$; (ii) $\mu_{\mathrm{Mo}} \leqslant$ $E(\mathrm{Mo}), \mu_{\mathrm{S}} \leqslant E(\mathrm{~S})$, and $\mu_{\mathrm{Mn}} \leqslant E(\mathrm{Mn})$ to avoid precipitation of Mo, S, and Mn, respectively; (iii) $\mu_{\mathrm{Mn}}+\mu_{\mathrm{S}} \leqslant E(\mathrm{MnS}$ ) to avoid precipitation of $\mathrm{MnS}$. We note that $\mathrm{Mn}$ richness can lead to the formation of $\mathrm{Mn}$ clusters or $\mathrm{MnS}$. $\mathrm{Mn}_{\mathrm{HT}}$ is always favorable over $\mathrm{Mn}_{\mathrm{MoT}}$. The results in Fig. 2 indicate that $\mathrm{Mn}_{\mathrm{S}}$ is favorable in an Mo rich environment and $\mathrm{Mn}_{\mathrm{Mo}}$ in an $\mathrm{S}$ rich environment.

We first study the interaction between the Mn dopants and $\mathrm{MoS}_{2}$ by means of a Löwdin charge analysis. The total magnetic moment of the $\mathrm{Mn}_{\mathrm{Mo}}$ system is $1.00 \mu_{B}$, corresponding 
(a)

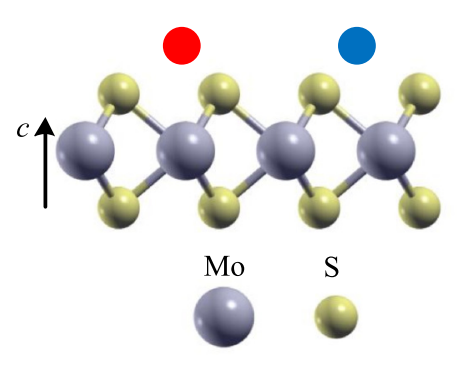

(b)

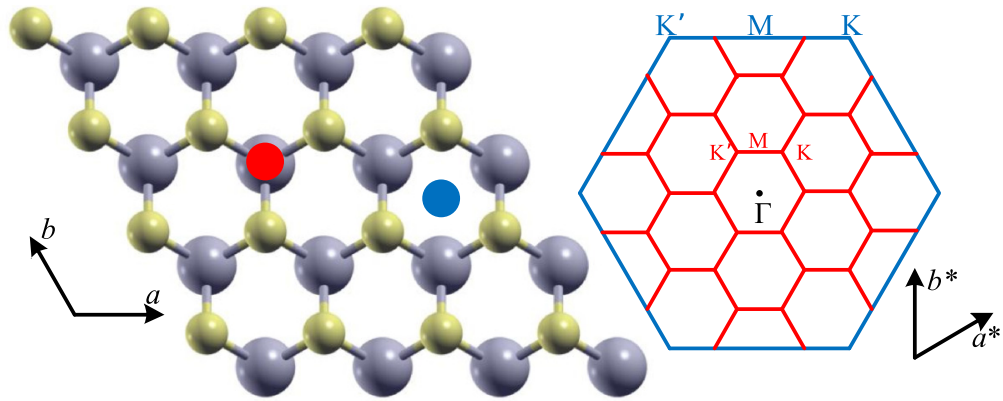

FIG. 1. (Color online) (a) Side view and (b) top view of single-layer $\mathrm{MoS}_{2}$ for a $4 \times 4$ supercell. The red and blue circles represent Mn doping on top of Mo and on top of the hollow site, respectively. (c) Brillouin zone corresponding to the $1 \times 1$ unit cell (blue) and $4 \times 4$ supercell (red), where the $K$ and $K^{\prime}$ points of the unit cell, respectively, are folded onto the $K$ and $K^{\prime}$ points of the supercell.

to one excess $3 d$ electron. The fact that the sum of the absolute moments of all atoms is $1.89 \mu_{B}$ indicates that there is a strong magnetic coupling to the $\mathrm{MoS}_{2}$. The Mn moment amounts to $1.30 \mu_{B}$ (long-range ferromagnetic coupling), to which the moments of the neighboring $\mathrm{S}$ and Mo atoms are aligned antiferromagnetically [24,25]. For the $\mathrm{Mn}_{\mathrm{S}}$ system the total magnetic moment is $3.00 \mu_{B}$ and the sum of the absolute moments of all atoms is $6.10 \mu_{B}$. The Mn moment amounts to $4.30 \mu_{B}$ and the moments of its neighbors to $-0.43 \mu_{B}$. Moreover, the Mo atoms in the second coordination sphere have moments of $0.04 \mu_{B}$. We find that the hybridization between the $\mathrm{Mn}$ and neighboring Mo and $\mathrm{S}$ atoms is enhanced for the $\mathrm{Mn}_{\mathrm{S}}$ system as compared to the $\mathrm{Mn}_{\mathrm{Mo}}$ system. The total magnetic moment of the $\mathrm{Mn}_{\mathrm{HT}}$ system is $3.00 \mu_{B}$ and the sum of the absolute moments of all atoms is $4.51 \mu_{B}$. The Mn moment amounts to $3.68 \mu_{B}$ and we obtain for the nearest $\mathrm{S}$ and Mo atoms values of $-0.03 \mu_{B}$ and $-0.15 \mu_{B}$, respectively. Since the bond length of the absorbed atom to the adjacent $S$ atoms is only $2.15 \AA$, the Mn dopant is strongly chemisorbed.

The spin polarized total density of states for the $\mathrm{Mn}_{\mathrm{Mo}}$ system, see Fig. 3(a), shows that the $3 d$ states split into three

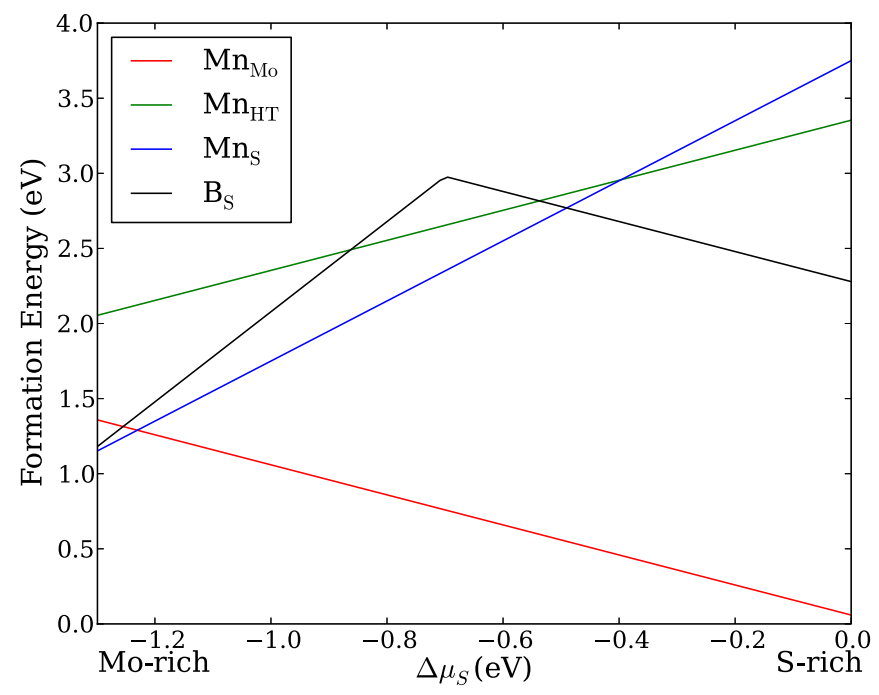

FIG. 2. (Color online) Formation energy for various impurities in $\mathrm{MoS}_{2}$ as a function of the $\mathrm{S}$ chemical potential. groups $\left(a, e_{1}\right.$, and $\left.e_{2}\right)$ in the trigonal prismatic environment of the chalcogen atoms. Because Mn has one valence electron more than Mo, this excess electron occupies the $a$ state, which explains the total magnetic moment of $1.00 \mu_{B}$. Opposite to the $\mathrm{Mn}_{\mathrm{Mo}}$ system, in the $\mathrm{Mn}_{\mathrm{S}}$ system, see Fig. 3(b), the energy of the valence band edge is lower for the spin-up than for the spin-down channel. We observe in Fig. 3(b) two defect states in the band gap, where the state near the valence band edge is due to the $\mathrm{Mn} 3 d_{x z / y z}$ and adjacent Mo $3 d$ orbitals and that near the conduction band edge is largely due to the Mo $3 d$ orbitals. There are three defect states found in the band gap for the $\mathrm{Mn}_{\mathrm{HT}}$ system, see Fig. 3(c). The two occupied spin-down states are due to the Mn $3 d$ and adjacent S $2 p$ and Mo $3 d$ orbitals, whereas the unoccupied spin-up state is largely due to the latter two orbitals. The energy of the valence band edge is higher for the spin up than for the spin-down channel.

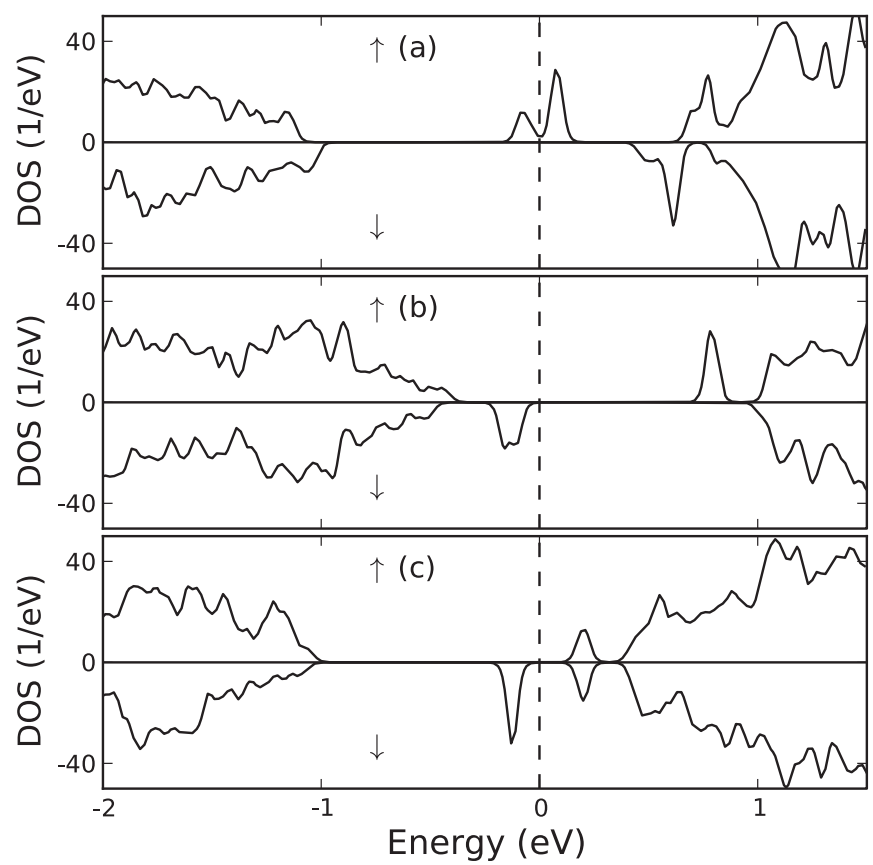

FIG. 3. Total spin polarized density of states (DOS) for Mn-doped single-layer $\mathrm{MoS}_{2}$ without spin orbit coupling. (a), (b), and (c) refer to the $\mathrm{Mn}_{\mathrm{Mo}}, \mathrm{Mn}_{\mathrm{S}}$, and $\mathrm{Mn}_{\mathrm{HT}}$ systems, respectively. 

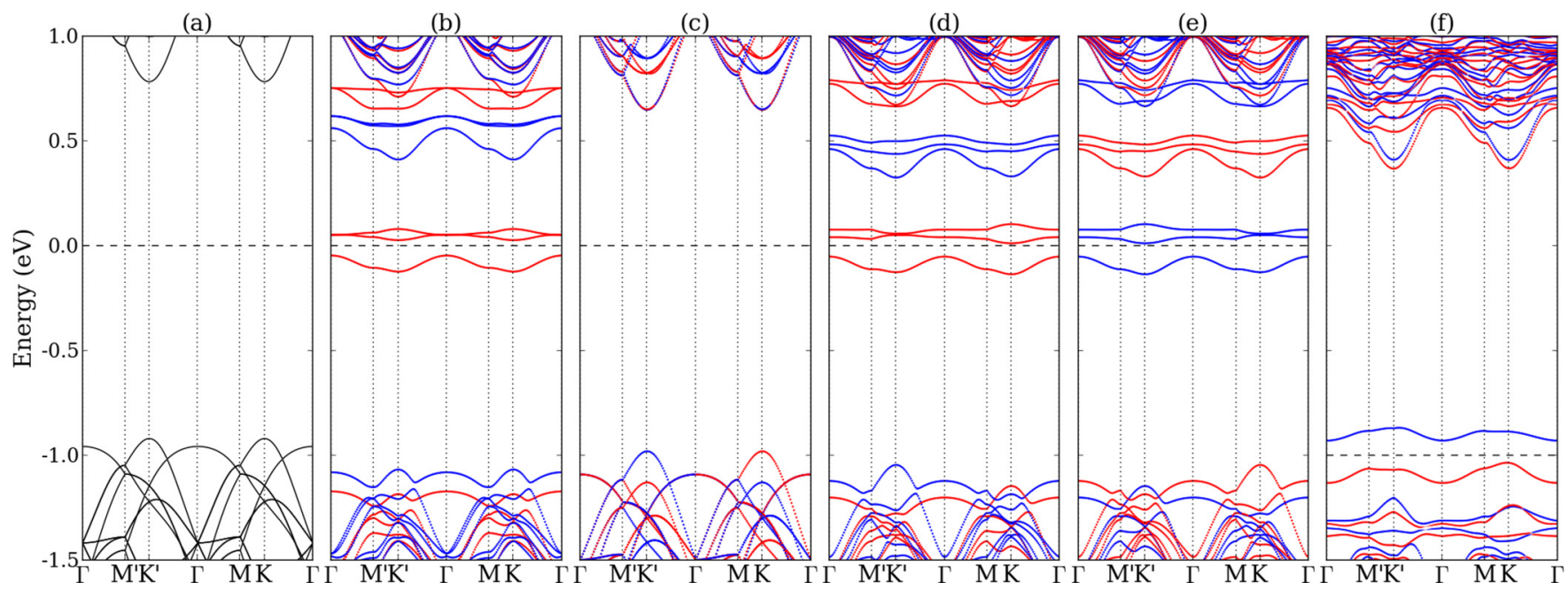

FIG. 4. (Color online) Electronic band structures for single-layer $\mathrm{MoS}_{2}$ : (a) without doping and without spin orbit coupling, (b) with Mn doping and without spin orbit coupling, (c) without doping and with spin orbit coupling, (d) with $\mathrm{Mn}_{\mathrm{Mo}}$ doping and with spin orbit coupling [inverted spin polarization in (e)], and (f) with $\mathrm{B}_{\mathrm{S}}$ doping and with spin orbital coupling. Red and blue color indicates spin up and down, respectively.

In the spin degenerate band structure of pristine single-layer $\mathrm{MoS}_{2}$ the valence band maxima and conduction band minima are located at the $K$ and $K^{\prime}$ points of the Brillouin zone, see Fig. 4(a). Results for the $\mathrm{Mn}_{\text {Mo }}$ system obtained without spin orbit coupling in Fig. 3(b) show for the highest valence band a splitting by $\Delta E^{v}=E_{\downarrow}^{v}-E_{\uparrow}^{v}=88 \mathrm{meV}$ at the $K$ and $K^{\prime}$ points, which is consistent with Fig. 3(a). $\Delta E^{v}$ equals -85 and $-38 \mathrm{meV}$ for the $\mathrm{Mn}_{\mathrm{S}}$ and $\mathrm{Mn}_{\mathrm{HT}}$ systems, respectively.

Taking into account the spin orbit coupling, see Figs. 4(c), 4(d), and 4(e), the momentum-dependent spin polarization is defined as the expectation value of the Pauli matrices

$$
m_{\alpha, i}(\mathbf{k})=\frac{1}{\Omega} \sum_{\sigma \sigma^{\prime}} \int \phi_{\mathbf{k}, i}^{\sigma^{\prime} *}(\mathbf{r}) \sigma_{\alpha}^{\sigma^{\prime} \sigma} \phi_{\mathbf{k}, i}^{\sigma}(\mathbf{r}) d \mathbf{r}
$$

where $\Omega$ is the volume of the system, $k$ is the wave vector, $\alpha$ denotes the Cartesian axis, $\phi_{\mathbf{k}, i}^{\sigma}(\mathbf{r})$ is the Kohn-Sham eigenspinor, and $\sigma_{\alpha}^{\sigma^{\prime} \sigma}$ represents the matrix elements of the Pauli spin operator. The band structure of pristine single-layer $\mathrm{MoS}_{2}$ is characterized by a giant spin splitting of $148 \mathrm{meV}$ at the $K$ and $K^{\prime}$ points, because there is no inversion symmetry [11]. The valence band edge at the $K / K^{\prime}$ point is due to spin-up/down states, whereas the situation is inverted for the conduction band edge. As a consequence, the exchange interaction between $\mathrm{MoS}_{2}$ and a magnetic dopant will enhance/reduce the splitting at the $K / K^{\prime}$ point. Taking the $\mathrm{Mn}_{\text {Mo }}$ system as an example, the band structure in Fig. 4(d) shows a spin-down valence band valley at the $K^{\prime}$ point and a comparison to Fig. 4(c) confirms valley polarization for the valence band. We next demonstrate that the valley polarization can be controlled by the spin polarization. When the spin direction is inverted, see Fig. 4(e), the character changes to spin up and the location to the $K$ point, i.e., the valley polarization is inverted. For the $\mathrm{Mn}_{\mathrm{S}}$ and $\mathrm{Mn}_{\mathrm{HT}}$ systems the same dependence is observed, only the spin direction is inverted from the beginning. The impurity states seen in Figs. 4(b), 4(d), and 4(e) are occupied by the extra valence electron of Mn. Since they are far away from the valence band maxima, they have no influence on the valley polarization.

These results can be understood from the effective Hamiltonian $\hat{H}=\hat{H}_{0}+\hat{H}_{1}+\hat{H}_{2}$ [15] for the $K$ and $K^{\prime}$ valleys, with

$$
\begin{gathered}
\hat{H}_{0}=a t\left(\tau k_{x} \hat{\sigma}_{x}+k_{y} \hat{\sigma}_{y}\right)+\frac{\Delta}{2} \hat{\sigma}_{z}, \\
\hat{H}_{1}=-\lambda \tau \frac{\hat{\sigma}_{z}-1}{2} \hat{s}_{z}, \\
\hat{H}_{2}=-x J_{\text {eff }}\langle S\rangle \hat{s}_{z} .
\end{gathered}
$$

Here, $\hat{H}_{0}$ is the Hamiltonian for the pristine single layer without spin orbit coupling and $a, t, \Delta, \lambda$, and $\tau$ denote the lattice constant, effective hopping integral, energy gap, spin splitting, and valley index, respectively. Moreover, $\hat{\sigma}$ and $\hat{s}$ are vectors of Pauli matrices. For $\mathrm{MoS}_{2}$ we have $a=3.193 \AA, t=1.10 \mathrm{eV}, \Delta=1.66 \mathrm{eV}$, and $2 \lambda=0.15 \mathrm{eV}$. For $\hat{H}=\hat{H}_{0}$ the spin-up and -down bands are degenerate at the $K$ and $K^{\prime}$ points, see Fig. 5(a). From the Zener model $[34,35]$ the effect of magnetic doping is given by $\hat{H}_{2}$, where $\Delta E=x J_{\text {eff }}\langle S\rangle$ with the dopant concentration $x$, the exchange constant $J_{\text {eff }}$, and the local magnetic moment $2\langle S\rangle$. For $\hat{H}=\hat{H}_{0}+\hat{H}_{2}$ with $\Delta E=-0.05 \mathrm{eV}$ the spin splitting induced by the magnetic doping has the same direction in both valleys, see Fig. 5(b). For $\hat{H}=\hat{H}_{0}+\hat{H}_{1}$ the spins are inverted at the $K$ and $K^{\prime}$ points, see Fig. 5(c). Combining all three terms of the Hamiltonian the spin splitting at the $K$ and $K^{\prime}$ points amounts to $2 \lambda-\Delta E$ and $-2 \lambda-\Delta E$, respectively, which couples the valley polarization to the spin polarization. Figs. 5(d)/5(e) show this situation for $\Delta E=-/+0.05 \mathrm{eV}$, consistent with Figs. 4(d)/4(e).

The spin splitting may be tuned via the strength $2 \lambda$ of the spin orbit coupling, e.g., from 0.15 to $0.46 \mathrm{eV}$ from $\mathrm{MoS}_{2}$ to $\mathrm{WSe}_{2}$. Concerning $\Delta E$, our first-principles calculations yield exchange constants $J_{\text {eff }}$ of $2.17,-0.63$, and $-0.33 \mathrm{eV}$, respectively, for the $\mathrm{Mn}_{\mathrm{Mo}}, \mathrm{Mn}_{\mathrm{S}}$, and $\mathrm{Mn}_{\mathrm{HT}}$ systems with a $6.25 \%$ dopant concentration. Different doping sites result not 
(a)
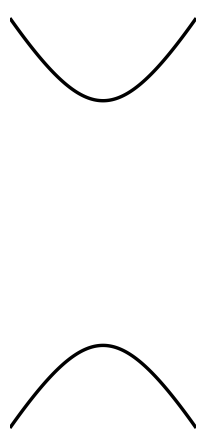

$\mathrm{K}, \mathrm{K}^{\prime}$ (b)
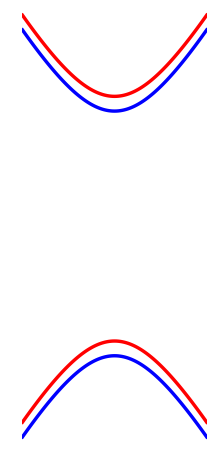

$\mathrm{K}, \mathrm{K}^{\prime}$ (c)

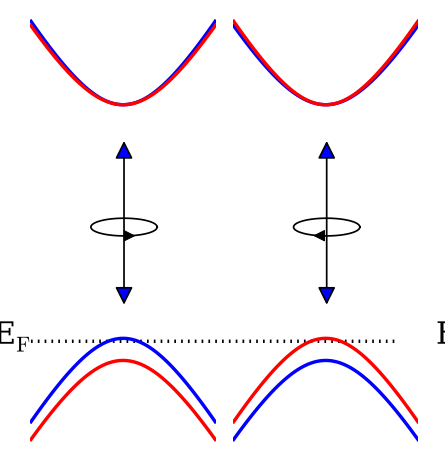

$K^{\prime}$
$\mathrm{K}$ (d)
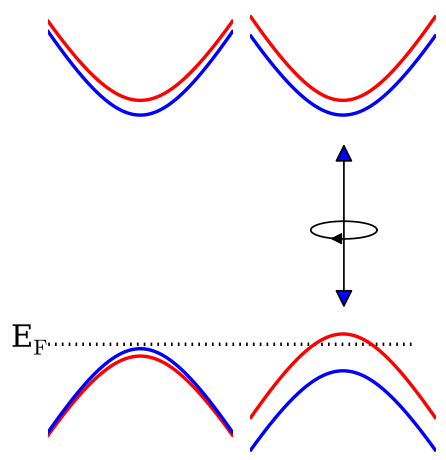

$\mathrm{K}^{\prime}$
K (e)

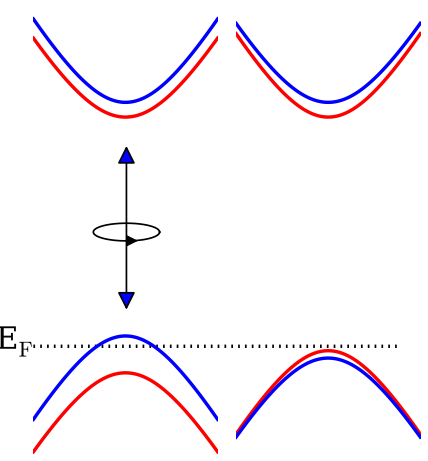

$K^{\prime}$

K

FIG. 5. (Color online) Schematic view of the band structure of single-layer $\operatorname{MoS}_{2}$ near the $K$ and $K^{\prime}$ points: (a) pristine without spin orbit coupling, (b) with exchange field and without spin orbit coupling, (c) pristine with spin orbit coupling, and (d) with exchange field and with spin orbit coupling. (e) Same as (d) but with inverted spin polarization.

only in different local magnetic moments but also in different exchange constants. Therefore, it is important to control the doping site, because otherwise positive and negative exchange constants could compensate each other. For example, for Mn deposition by evaporation [31] the $\mathrm{Mn}_{\mathrm{S}}$ and $\mathrm{Mn}_{\mathrm{HT}}$ configurations are realized, which correspond to negative exchange constants. A higher dopant concentration $x$ will enhance $\Delta E$, where the $\mathrm{Mn}_{\mathrm{Mo}}$ system is reported to support concentrations up to $x=15 \%$ while remaining ferromagnetic [24]. Calculations for this system using $x=12.5 \%$ and two different spatial arrangements of the Mn atoms confirm the ferromagnetic state with a total magnetic moment of $2.00 \mu_{B}$. The valley polarization persists in both cases, which indicates that neither the dopant concentration nor disorder affect our conclusions. While we consider only Mn doping in $\mathrm{MoS}_{2}$, other transition metals, such as $\mathrm{V}, \mathrm{Cr}, \mathrm{Fe}, \mathrm{Co}$, and $\mathrm{Ni}$, doped in $M X_{2}$ are also promising [23,36,37]. Similar to the observation that EuO introduces spin polarization in graphene by the proximity effect [38], we note that valley polarization may be achieved in $M X_{2}$ on ferromagnetic substrates, such as EuO and EuS.

Control of the valley polarization by magnetic doping paves the way to valleytronics. Because the spin splitting is stronger in the valence band valleys than in the conduction band valleys, the valley polarization will be more pronounced in the valence than in the conduction band. Therefore, hole doped $M X_{2}$ probably is more promising for applications as in this case a spin and valley polarized current can be obtained. Mn doping alone does not bring the Fermi level close to the valence band so that additional hole doping is required, which can be achieved following Refs. $[39,40]$. From a fabrication point of view, such codoping is complicated and difficult to control. However, it is predicted that ferromagnetism can be achieved by the substitution of $\mathrm{S}$ by nonmetal elements, such as $\mathrm{H}, \mathrm{B}$, $\mathrm{N}$, and $\mathrm{F}$, among which $\mathrm{B}$ and $\mathrm{N}$ induce hole doping with a magnetic moment of $1 \mu_{B}$ [41]. In this reference it also has been demonstrated that $\mathrm{B}_{\mathrm{S}}$ is the most stable configuration. In order to avoid the precipitation of $\mathrm{BS}_{2}, \mathrm{~B}_{2} \mathrm{~S}_{3}, \mathrm{MoB}$, and $\mathrm{MoB}_{2}$ the relations $\mu_{\mathrm{B}}+2 \mu_{\mathrm{S}} \leqslant E\left(\mathrm{BS}_{2}\right), 2 \mu_{\mathrm{B}}+3 \mu_{\mathrm{S}} \leqslant E\left(\mathrm{~B}_{2} \mathrm{~S}_{3}\right)$, $\mu_{\mathrm{Mo}}+\mu_{\mathrm{B}} \leqslant E(\mathrm{MoB})$, and $\mu_{\mathrm{Mo}}+2 \mu_{\mathrm{B}} \leqslant E\left(\mathrm{MoB}_{2}\right)$ must be satisfied. Figure 2 indicates that $\mathrm{B}_{\mathrm{S}}$ is favorable in an Mo rich environment. For an increased concentration of $6.25 \%$ the system remains ferromagnetic. The band structure for a $3.125 \% \mathrm{~B}_{\mathrm{S}}$ concentration (which can be obtained by plasma treatment $[42,43]$ ) is shown in Fig. 4(f), indicating that valley polarization is achieved. In addition, circularly polarized photons can be generated because of the coupling of the valley and spin polarizations. By inverting the spin polarization the polarization direction of the photon can be switched. Single-layer $\mathrm{MX}_{2}$ is a prototypical two-dimensional honeycomb lattice material with strong spin orbit coupling and a broken inversion symmetry. Perpendicular spins with opposite direction at the $K$ and $K^{\prime}$ points have also been reported for other systems, such as the Tl/Si(111) surface alloy [44-48]. We therefore expect that a valley polarization can be achieved in magnetically doped $\mathrm{Tl} / \mathrm{Si}(111)$ and related systems.

In conclusion, we predict that the valley polarization in single-layer semiconducting $M X_{2}$ can be controlled by magnetic doping. Studying Mn doping at various sites in singlelayer $\mathrm{MoS}_{2}$ as an example, we have analyzed the effects on the electronic structure by first-principles calculations and have explained the results by means of a low-energy effective Hamiltonian. We find that the valley polarization depends strongly on the strength of the spin orbit coupling and the exchange interaction, which varies with the doping site and dopant concentration to enable an effective control. Circularly polarized photons can be achieved because of spin-valley coupling. The results are expected to be valid also for other two-dimensional honeycomb lattice systems with strong spin orbit coupling and without inversion symmetry.

We thank M. Tahir for helpful discussion. This work has been supported by a KAUST CRG grant and computational resources have been provided by KAUST IT.
[1] S. A. Wolf, D. D. Awschalom, R. A. Buhrman, J. M. Daughton, S. von Molnar, M. L. Roukes, A. Y. Chtchelkanova, and D. M. Treger, Science 294, 1488 (2001).
[2] O. Gunawan, Y. P. Shkolnikov, K. Vakili, T. Gokmen, E. P. De Poortere, and M. Shayegan, Phys. Rev. Lett. 97, 186404 (2006). 
[3] Y. P. Shkolnikov, E. P. De Poortere, E. Tutuc, and M. Shayegan, Phys. Rev. Lett. 89, 226805 (2002).

[4] Z. W. Zhu, A. Collaudin, B. Fauqué, W. Kang, and K. Behnia, Nat. Phys. 8, 89 (2012).

[5] M. Kohda, T. Bergsten, and J. Nitta, J. Phys. Soc. Jpn. 77, 031008 (2008).

[6] S. LaShell, B.A. McDougall, and E. Jensen, Phys. Rev. Lett. 77, 3419 (1996).

[7] K. S. Novoselov, A. K. Geim, S. V. Morozov, D. Jiang, Y. Zhang, S. V. Dubonos, I. V. Grigorieva, and A. A. Firsov, Science 306, 666 (2004).

[8] M. Z. Hasan and C. L. Kane, Rev. Mod. Phys. 82, 3045 (2010).

[9] A. Splendiani, L. Sun, Y. Zhang, T. Li, J. Kim, C.-Y. Chim, G. Galli, and F. Wang, Nano Lett. 10, 1271 (2010).

[10] K. F. Mak, C. Lee, J. Hone, J. Shan, and T. F. Heinz, Phys. Rev. Lett. 105, 136805 (2010).

[11] Z. Y. Zhu, Y. C. Cheng, and U. Schwingenschlögl, Phys. Rev. B 84, 153402 (2011).

[12] Y. C. Cheng, Z. Y. Zhu, M. Tahir, and U. Schwingenschlögl, Europhys. Lett. 102, 57001 (2013).

[13] D. Xiao, W. Yao, and Q. Niu, Phys. Rev. Lett. 99, 236809 (2007).

[14] W. Yao, D. Xiao, and Q. Niu, Phys. Rev. B 77, 235406 (2008).

[15] D. Xiao, G.-B. Liu, W. X. Feng, X. D. Xu, and W. Yao, Phys. Rev. Lett. 108, 196802 (2012).

[16] H. L. Zeng, J. F. Dai, W. Yao, D. Xiao, and X. D. Cui, Nat. Nanotechnol. 7, 490 (2012).

[17] K. F. Mak, K. L. He, J. Shan, and T. F. Heinz, Nat. Nanotechnol. 7, 494 (2012).

[18] T. Cao, G. Wang, W. P. Han, H. Q. Ye, C. R. Zhu, J. R. Shi, Q. Niu, P. H. Tan, E. G. Wang, B. L. Liu, and J. Feng, Nat. Commun. 3, 887 (2012).

[19] X.-L. Qi, R. D. Li, J. D. Zang, and S.-C. Zhang, Science 323, 1184 (2009).

[20] R. Yu, W. Zhang, H.-J. Zhang, S.-C. Zhang, X. Dai, and Z. Fang, Science 329, 61 (2010).

[21] I. Garate and M. Franz, Phys. Rev. Lett. 104, 146802 (2010).

[22] Z. H. Qiao, S. Y. A. Yang, W. X. Feng, W.-K. Tse, J. Ding, Y. G. Yao, J. Wang, and Q. Niu, Phys. Rev. B 82, 161414(R) (2010).

[23] Y. C. Cheng, Z. Y. Zhu, W. B. Mi, Z. B. Guo, and U. Schwingenschlögl, Phys. Rev. B 87, 100401(R) (2013).

[24] A. Ramasubramaniam and D. Naveh, Phys. Rev. B 87, 195201 (2013).

[25] R. Mishra, W. Zhou, S. J. Pennycook, S. T. Pantelides, and J.-C. Idrobo, Phys. Rev. B 88, 144409 (2013).

[26] P. Giannozzi et al., J. Phys.: Condens. Matter 21, 395502 (2009).

[27] D. Vanderbilt, Phys. Rev. B 41, 7892 (1990).

[28] T. Jungwirth, J. Sinova, J. Mašek, J. Kučera, and A. H. MacDonald, Rev. Mod. Phys. 78, 809 (2006).
[29] R. F. Frindt, A. S. Arrott, A. E. Curzon, B. Heinrich, S. R. Morrison, T. L. Templeton, R. Divigalpitiya, M. A. Gee, P. Joensen, P. J. Schurer, and J. L. LaCombe, J. Appl. Phys. 70, 6224 (1991).

[30] M. Kamaratos and C. A. Parageorgopoulos, Surf. Sci. 160, 451 (1985).

[31] J. R. Lince, T. B. Stewart, P. D. Fleischauer, J. A. Yarmoff, and A. Taleblbrahimi, J. Vac. Sci. Tech. A 7, 2469 (1989).

[32] X. Zhong, H. D. Yang, S. J. Guo, S. W. Li, G. L. Gou, Z. Y. Niu, Z. P. Dong, Y. J. Lei, J. Jin, R. Li, and J. T. Ma, J. Mater. Chem. 22, 13925 (2012).

[33] F. L. Deepak, R. Esparza, B. Borges, X. Lopez-Lozano, and M. Jose-Yacaman, ACS Catal. 1, 537 (2011).

[34] T. Dietl, H. Ohno, F. Matsukura, J. Cibert, and D. Ferrand, Science 287, 1019 (2000).

[35] S. Sanvito, P. Ordejón, and N. A. Hill, Phys. Rev. B 63, 165206 (2001).

[36] Y. G. Zhou, Q. L. Su, Z. G. Wang, H. Q. Deng, and X. T. Zu, Phys. Chem. Chem. Phys. 15, 18464 (2013).

[37] H. L. Shi, H. Pan, Y.-W. Zhang, and B. I. Yakobson, Phys. Rev. B 88, 205305 (2013).

[38] H. X. Yang, A. Hallal, D. Terrade, X. Waintal, S. Roche, and M. Chshiev, Phys. Rev. Lett. 110, 046603 (2013).

[39] M. Laskar, D. N. Nath, L. Ma, E. Lee, C. H. Lee, T. Kent, Z. Yang, R. Mishra, M. A. Roldan, J.-C. Idrobo, S. T. Pantelides, S. J. Pennycook, R. Myers, Y. Wu, and S. Rajan, arXiv:1310.6494.

[40] K. Dolui, I. Rungger, C. Das Pemmaraju, and S. Sanvito, Phys. Rev. B 88, 075420 (2013).

[41] Q. Yue, S. L. Chang, S. Q. Qin, and J. B. Li, Phys. Lett. A 377, 1362 (2013).

[42] M. Chen, H. Nam, S. Wi, L. Ji, X. Ren, L. Bian, S. Lu, and X. Liang, Appl. Phys. Lett. 103, 142110 (2013).

[43] M. Chen, H. Nam, S. Wi, G. Priessnitz, I. Gunawan, and X. Liang, ACS Nano, doi:10.1021/nn501181t (2014).

[44] K. Sakamoto, T. Oda, A. Kimura, K. Miyamoto, M. Tsujikawa, A. Imai, N. Ueno, H. Namatame, M. Taniguchi, P. E. J. Eriksson, and R. I. G. Uhrberg, Phys. Rev. Lett. 102, 096805 (2009).

[45] M.-H. Liu and C.-R. Chang, Phys. Rev. B 80, 241304(R) (2009).

[46] J. Ibañez-Azpiroz, A. Eiguren, and A. Bergara, Phys. Rev. B 84, 125435 (2011).

[47] K. Sakamoto, T.-H. Kim, T. Kuzumaki, B. Müller, Y. Yamamoto, M. Ohtaka, J. R. Osiecki, K. Miyamoto, Y. Takeichi, A. Harasawa, S. D. Stolwijk, A. B. Schmidt, J. Fujii, R. I. G. Uhrberg, M. Donath, H. W. Yeom, and T. Oda, Nat. Commun. 4, 2073 (2013).

[48] S. D. Stolwijk, A. B. Schmidt, M. Donath, K. Sakamoto, and P. Krüger, Phys. Rev. Lett. 111, 176402 (2013). 